\title{
The Technology of Collecting Initial Data for Constructing Models for Assessing the Functional State of a Person by Pupillary Response to Changes In Illumination
}

\author{
Oleg Yuryevich Panischev ${ }^{1}$, Rufat Faig Ogly Babayev ${ }^{1}$, Dmitriy Gennadievich Petrosyants ${ }^{2}$, Alexey Sergeevich Katasev ${ }^{2}$, \\ Amir Muratovich Akhmetvaleev ${ }^{2}$, Irina Vladislavovna Akhmetvaleeva ${ }^{2}$, Dina Vladimirovna Kataseva ${ }^{2}$ \\ ${ }^{1}$ Researcher of the Research Laboratory of Near Space Research of the Kazan Federal University, Russia. \\ Scopus ID: 8355604900; ORCID: 0000-0001-5490-912X, \\ ${ }^{2}$ Junior Researcher of the Research Laboratory of Near Space Research of the Kazan Federal University, Russia. \\ Scopus ID: n/a; ORCID: 0000-0003-4894-8354, \\ ${ }^{3}$ graduate student of the Department of Information Security Systems of the Institute of Computer Technologies and Information \\ Protection of the Kazan National Research Technical University named after A.N. Tupolev-KAI, Russia.
}

Scopus ID: 57205544513; ORCID: 0000-0002-7543-3391,

${ }^{4}$ Doctor of Technical Sciences, Professor of the Department of Information Security Systems of the Institute of Computer Technologies and Information Security of the Kazan National Research Technical University named after A.N. Tupolev-KAI, Russia. Scopus ID: 57193408902; ORCID: 0000-0002-9446-0491,

${ }^{5}$ Candidate of Technical Sciences, Associate Professor of the Department of Information Security Systems of the Institute of Computer Technologies and Information Security of the Kazan National Research Technical University named after A.N.

Tupolev-KAI, Russia. Scopus ID: 57202913457; ORCID: 0000-0003-0384-9539,

${ }^{6}$ Master student of the Department of Information Security Systems of the Institute of Computer Technologies and Information Protection of the Kazan National Research Technical University named after A.N. Tupolev-KAI, Russia.

Scopus ID: n/a; ORCID: 0000-0001-7775-903X,

${ }^{7}$ Senior Lecturer of the Department of Information Security Systems of the Institute of Computer Technologies and Information Protection of the Kazan National Research Technical University named after A.N. Tupolev-KAI, Russia.

Scopus ID: 57193401954; ORCID: 0000-0001-6141-8329,

\begin{abstract}
This article solves the problem of collecting initial data for constructing models for assessing the functional state of a person by pupillary response to changes in illumination. We analyzed the drawbacks of the traditional approach to collecting initial data using computer vision and time series smoothing methods. Attention is focused on the importance of the quality of the initial data for the creation of adequate highprecision mathematical models. We actualized the need for manual marking of the iris and pupil circles to improve the accuracy and quality of the initial data. We described the initial data collection stages in the proposed technology. We gave an example of the resulting pupillogram, which has a smooth shape and does not contain outliers, noise, anomalies and missing values. Based on the given technology, we developed a software and hardware complex, which is a collection of specially developed software that has two main modules and hardware implemented on the basis of a Raspberry Pi 4 Model B microcomputer, with peripheral equipment that implements the specified functionality. To evaluate the effectiveness of the given technology for collecting initial data, we used models of a single-layer perspetron and a collective of neural networks, together with the initial data on the functional state of intoxication of a
\end{abstract}

person. The studies have shown that the number of errors of the 1 st and 2 nd genus in determining the assessment of the functional state of a person is lower, and the classification accuracy is higher when using the initial data generated by manual marking of circles, compared with the initial data collected by computer vision methods. Thus, the given technology for collecting initial data can be effectively used to build models for assessing the functional state of a person by pupillary response to changes in illumination.

Keywords: pupillometry, collection of initial data, computer vision, manual data marking, assessment of the functional state of a person, pupillary response.

\section{INTRODUCTION}

The pupillometry method is an objective method for diagnosing abnormalities in the functioning of the central and peripheral nervous systems of the human body [1-3]. The method is based on non-invasive and non-contact recording the pupil reaction of the human eye to a light stimulus using special video-pupillograph devices. In turn, the pupil reaction is presented in the form of a pupillogram - a time series reflecting the pupil area at each moment of time, during video recording. The subsequent analysis of the data obtained - the 
pupillogram values, - allows judging with a high degree of accuracy and reliability of the presence of various deviations in the functional state (FS) and human health in general.

With the development of technical means of computer vision, the use of the pupillometry method in assessing functional states seems to be highly promising not only in medical diagnostics systems, but also in public and transport security systems [4], as well as life and labor safety [5, 6]. In particular, the improvement of computer vision methods makes it possible to move away from the use of video pupillograph devices, which are usual for medical institutions, as well as use the inexpensive high-resolution video cameras and the corresponding software to assess functional states [7, 8]. This approach can insignificantly reduce the accuracy of FS assessment, but it can be used for screening diagnostics of a large number of people due to a significant reduction in material and technical costs.

At the same time, there is a factor that significantly complicates wide pupillometry in solving problems of FS assessment for various subject areas and applied problems not related to medical laboratory diagnostics: the prospects for the development of new effective mathematical models for FS assessment by pupil response are significantly limited by the framework of research teams and researchers with access to the expensive specialized equipment. This is due to the fact that building models requires high-quality raw data, the production of which depends on the hardware. In addition, the use of inexpensive video cameras to collect initial data is also hindered by the drawbacks of existing computer vision methods (eye image processing), which do not provide high accuracy in the selection of the desired circle contours, including low detail of the video image, and depending on the image distortions [9-11].

In this paper, in order to overcome high material and technical costs and data distortion, as a result of automatic computer processing of the eye image, we proposed a new technology for collecting initial data to build adequate models for FS assessment.

\section{METHODS}

The process of obtaining initial data - pupillograms reflecting the dynamics of changes in the pupil size to changes in illumination - is inextricably linked with such procedures as video recording of human eyes at the time of pupillary reaction, as well as subsequent analysis of images of human eyes to search for the circles of the pupil and iris [5, 12].

When using traditional pupillographic complexes, the video recording procedure is complicated by additional stages of adjusting the eyes in the video camera focus and eliminating the interferences $[5,12]$, where errors and inaccuracies can cause additional failures in the operation of computer vision algorithms used to isolate the boundaries of the desired circles of the pupil and iris. Pupillograms obtained using computer vision methods shall be additionally cleaned and smoothed, as shown in the following example.

As can be seen, the smoothed pupillogram (Fig. 1b) is smoother due to the elimination of noise, anomalies and missing values, compared to the original one (Fig. 1a). However, when smoothing time series, the series properties important for analysis are often lost, which can cause classification errors in the models built on their basis. This circumstance is unacceptable when it is necessary to build adequate systems for expert diagnostics and assessments. Thus, when collecting initial data, it is important to use approaches that minimize errors and improve the quality of the collected samples.

It is known that the manual data markup is often used when building various neural network models. For example, manually marked images of objects that shall be recognized by a neural network. Such data can significantly increase the quality of the initial data, but also increase the preparation time. At the same time, such an approach is necessary and justified in the construction of high-precision mathematical models. Therefore, to collect initial data (pupillograms), it is relevant to use the manual marking of images of the desired circles.

Based on the approaches of various methods of collecting the initial data [12-15], taking into account the proposed approach of manual marking, the developed new technology will consist of two main stages specified in Figure 2.
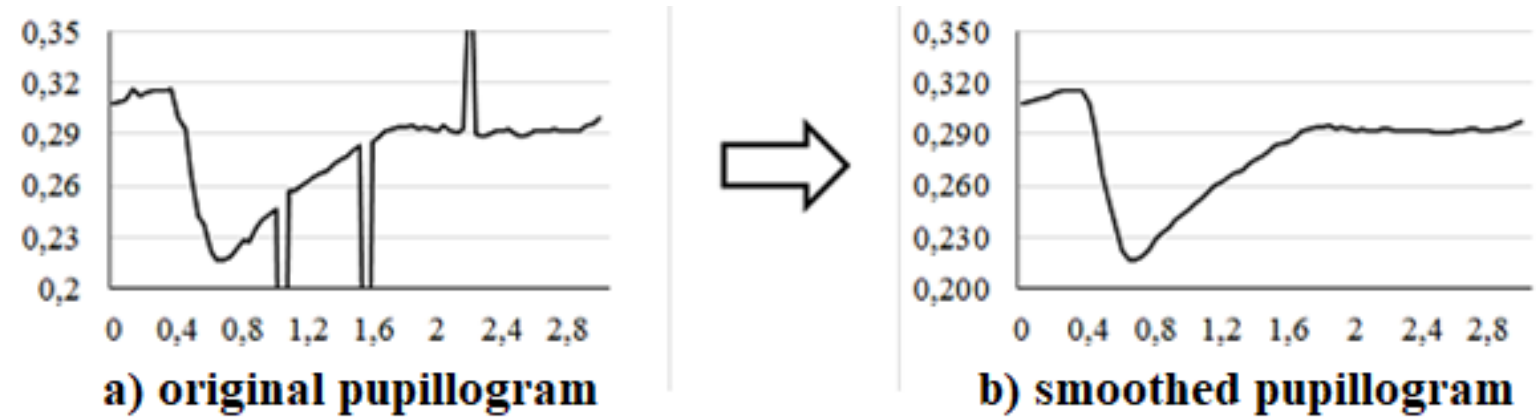

Figure 1. An example of the original (a) and smoothed (b) pupillograms 


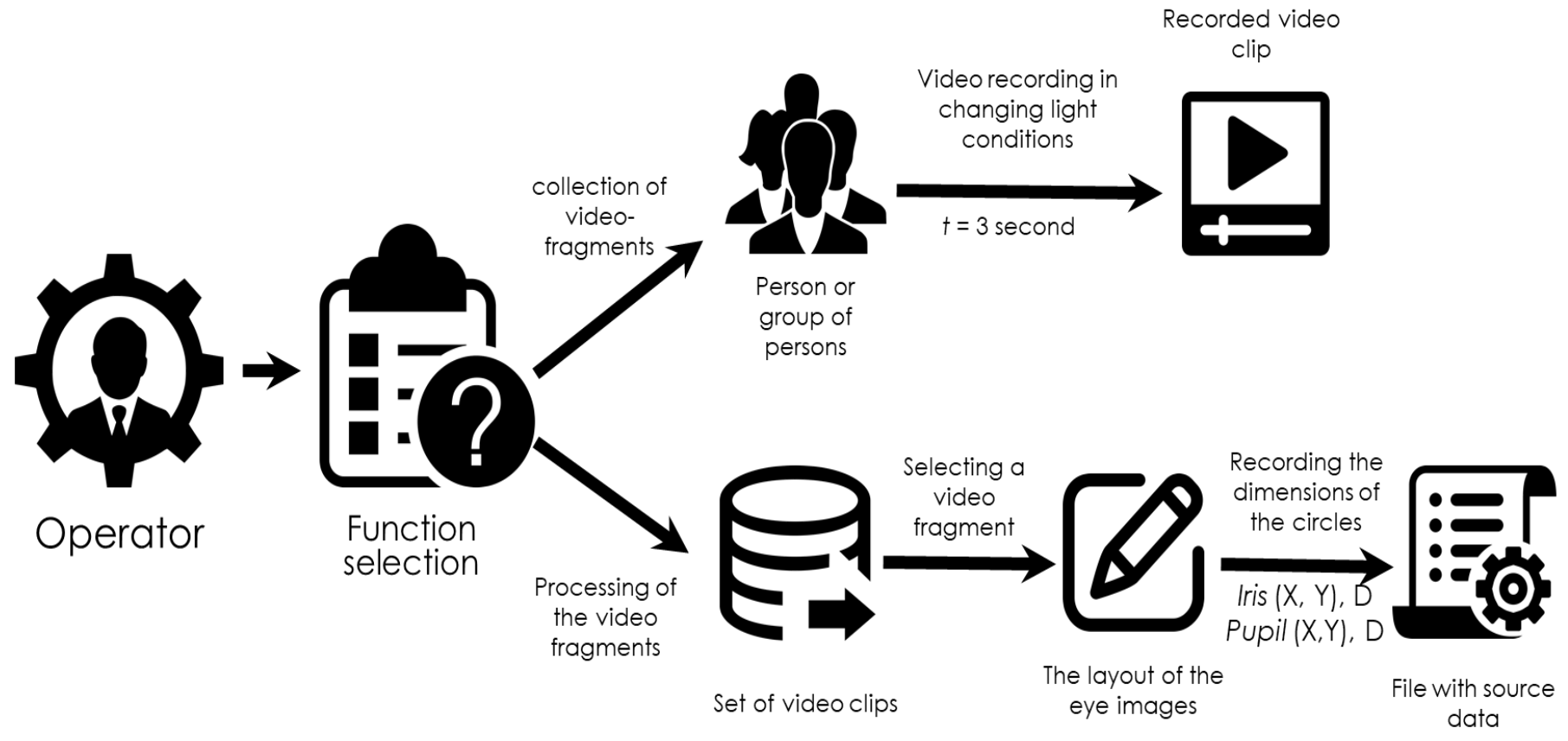

Figure 2. Scheme of initial data collection

The diagram shown in the figure reflects the sequence of the main stages of the developed technology. This scheme assumes a two-stage implementation of data collection: at the first stage, the operator collects video fragments, which allows making a large number of tests of pupillary reactions of people in a short time (the so-called screening); at the second stage, the recorded video fragments are processed manually by the operator to obtain data on the sizes of the pupil and iris. The set of data will be a time series reflecting the dynamics of changes in the pupil size relative to the iris size, i.e. pupillogram. The obvious advantage of this scheme lies in the speed of collecting video fragments, independence from expensive hardware laboratory complexes and special training of personnel, which minimizes costs, while obtaining a highquality sample of initial data to build adequate mathematical models.

When developing the technology, we introduced a number of conditions that determine the video fragment collection procedure:

- video fragment duration - 3 seconds;

- number of frames per second shall be equal to 25 ;

- video filming shall be carried out with the maximum resolution of the video camera, but not less than $1920 \times 1080$ pixels;

- the recorded video fragment shall be saved in a format that does not allow the quality loss in the received video data and the detail of video image;
- a person's face shall be in the view field of the camera;

- background lighting conditions - natural, not exceeding the average rate of artificial lighting;

- additional lighting conditions - the eyes shall be illuminated by a source of invisible infrared light;

- at the beginning of recording, a short-term (not more than $0.5 \mathrm{~s}$ ) light pulse of the visible spectrum with a brightness of $300 \mathrm{~cd} / \mathrm{m}^{2}$ is made towards the human eyes, causing a pupillary reaction;

The first technology stage consists in obtaining video fragments reflecting the pupillary reaction of the human eyes under conditions of changing illumination. Based on the existing requirements and conditions of the pupillometric examination, a 3 -second video recording is made during which the eye area shall be illuminated with an infrared light source, and a short-term supply of a bright light pulse shall be made at the beginning of recording.

To form a data sample of different classes of human FS, it is necessary to make at least 2 videos for each person, reflecting the differences in his/her FS: normal and pathological. Thus, the video clip collection stage is repeated at least twice the number of people involved in the initial data collection.

At the second technology stage, each fragment is manually marked with an indication of the location of the pupil and iris on the images. To do this, sequential actions are performed at each separate frame of the video fragment. Their execution scheme is shown in Figure 3. 


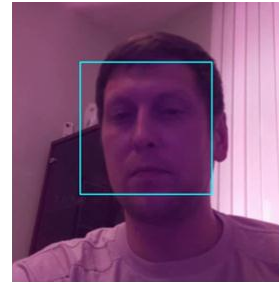

Searching for a face in a frame
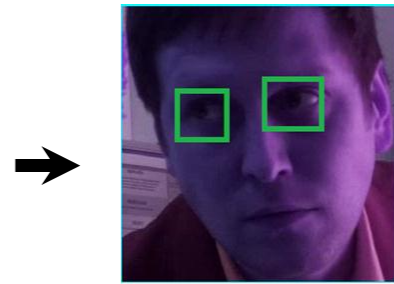

Search for eyes on a found face
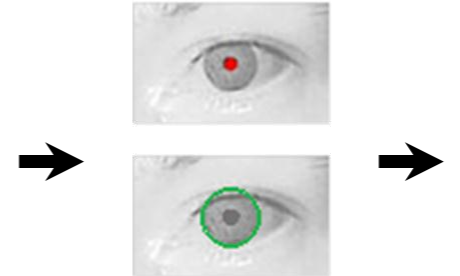

\section{Collecting the received data into} the metafile
Manual indication

of the centres of

the circles and

their diameters

Iris $(X, Y), D$

Pupil $(X, Y), D$

Figure 3. Scheme of eye image marking

The diagram shown in the figure consists of 4 sequential stages:

1) search for a face in the frame using the Viola-Jones computer vision method;

2) search for eyes in the face area using a similar method;

3) manual indication of the centers of the pupil and iris, indication of their diameters;

4) sequential storage of the received data.

The first and second stages consist in obtaining the eye image, for which the well-known Viola-Jones method [16], based on the Haar cascades, is used. A feature of its implementation is cascade (window) scanning of the image by rectangular regions with further object classification in the image. A set of matched cascades defines the boundaries of a person's face and eyes, thus allowing obtaining their approximate coordinates [17].

Thus, the operator shall manually specify the centers of the pupil and iris, as well as the sizes of their circles, expressed in diameters, at the third stage. In this case, it is important to make sure that the displayed data is correct by superimposing the constructed circles on the desired eye images. In addition, the inaccuracies of the Viola-Jones method used to detect the faces and eyes can lead to erroneous recognition of other areas. In this case, the operator shall mark this frame as a fragment that does not contain an eye image and not take it into account in the total data sample. The obtained circle coordinates and diameters are saved at the 4th stage sequentially frame-by-frame into the original data file of the processed video fragment.

Since the eye images can be of different sizes (pixel resolution), the absolute values of the pupil diameter cannot be used alone to collect the initial data. An additional parameter is needed to determine the relative pupil sizes in different images. This parameter is the iris diameter, since the iris size does not change over time, does not depend on illumination, and its size will be static. Therefore, to obtain homogeneous initial data, the relative pupil size will need to be calculated in normalized values using the formula:

$$
D=\frac{D_{\text {pupil }}}{D_{\text {iris }}},
$$

where $D_{\text {pupil }}$ - absolute pupil diameter, in pixels, $D_{\text {iris }}$ absolute iris diameter, in pixels After making calculations according to the given formula, we create a pupillogram, an example of which is shown in the following figure.

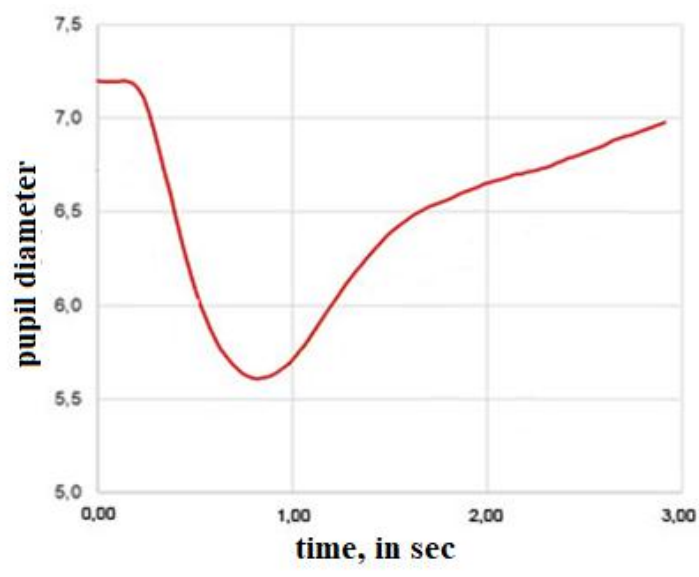

Figure 4. An example of a pupillogram

This pupillogram, due to manual marking of circles, has a smooth shape, does not contain outliers, noise, anomalous and missing values.

It should be noted that the obtained data (images with marked pupils and irises) can also be used to train neural network models of pattern recognition, for example, to train convolutional neural networks to automatically recognize pupils and irises in the eye images with validation of the existing manual markup. 
International Journal of Engineering Research and Technology. ISSN 0974-3154, Volume 13, Number 11 (2020), pp. 3616-3624

(C) International Research Publication House. https://dx.doi.org/10.37624/IJERT/13.11.2020.3616-3624

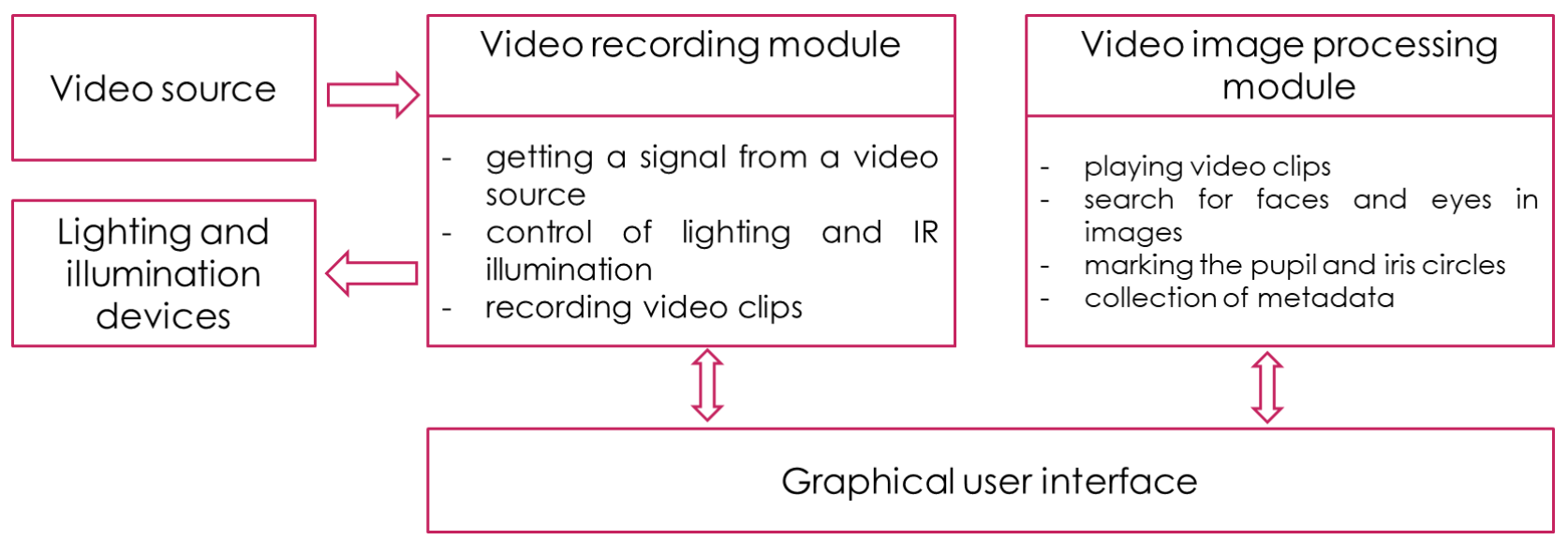

Figure 5. Software structure

\section{RESULTS AND DISCUSSION}

Based on the proposed technology for collecting initial data, we implemented a hardware-software complex (HSC). The software is based on a specially developed algorithm with the stages of proposed technology for collecting initial data: the collection of video fragments and manual marking of eye images.

The HSC is designed to implement the specified functionality of the software, namely, it includes a microcomputer designed to launch the program and display its interface, a source of illumination (infrared illumination and pulsed light) and other components. The set of software and hardware is a single hardware and software complex for collecting the initial data of the pupillary response to changes in illumination for further use in the tasks of constructing models to assess the functional state of a person.
The developed program has a modular structure and consists of two subroutines. Figure 5 shows its structural diagram.

The software consists of subprograms - modules: a module responsible for receiving and recording video images; a module responsible for processing recorded video images to obtain the initial data. The first module also controls all additional hardware equipment: turning on and receiving data from a video signal source; control of lighting (pulsed light source) and infrared illumination. The second module is responsible for processing the received data, namely: video playback; search for faces and eyes on the video sequence images with subsequent saving of data into separate files; marking of the pupil and iris circles of the human eye.

The hardware is a combination of various source components (Raspberry Pi 4 Model B microcomputer, video camera, flash and infrared light). The connection diagram of the original components in the device is shown in the following figure.

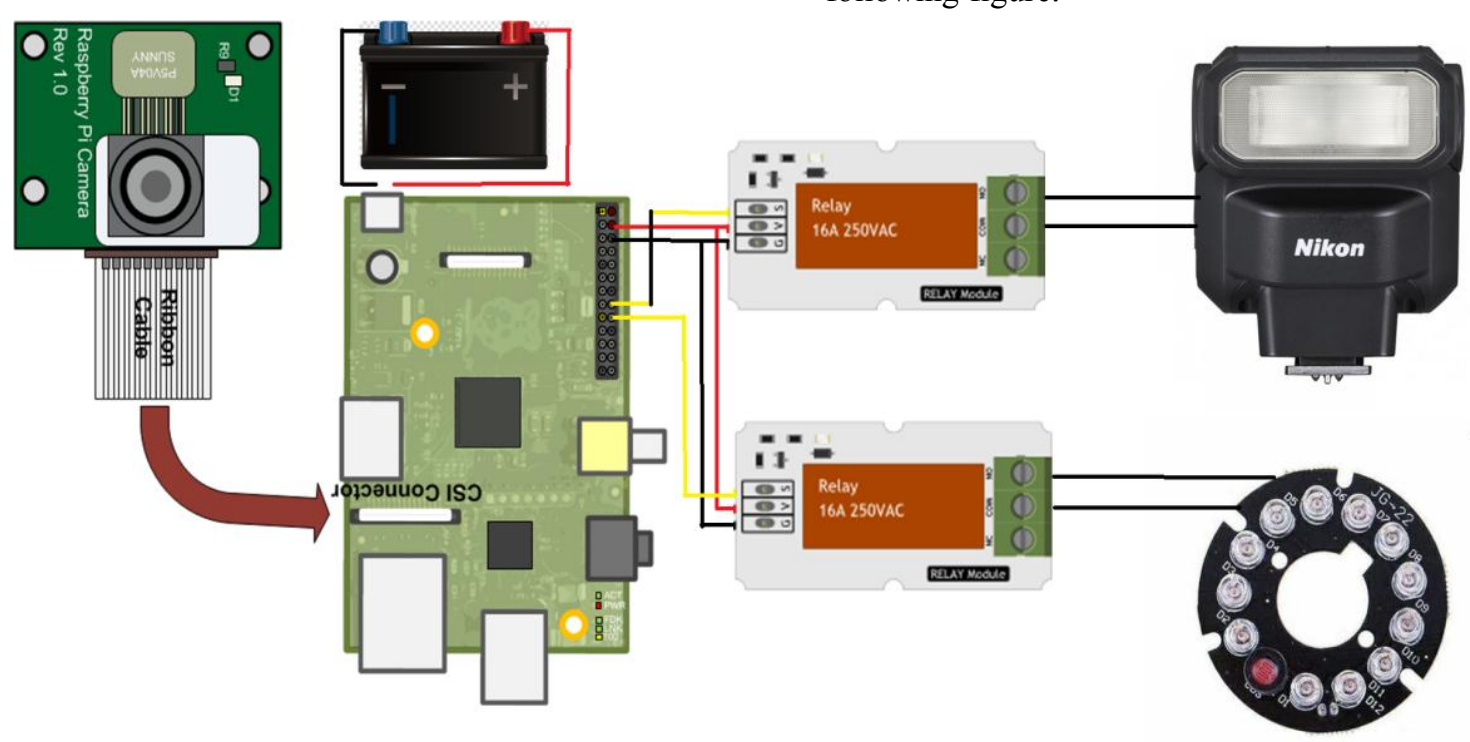

Figure 6. Hardware connection diagram 
The presented schematic diagram of the connection of the initial components of the developed device shows the relative simplicity of the hardware implementation and, as a result, characterizes the given solution as a highly economical and efficient hardware platform for collecting initial data. The developed hardware is assembled from components that are freely available in many retail chains specializing in radio electronics and microcomputers. The approximate cost of the device received is not more than $\$ 200$.

The effectiveness of the developed technology and HSC is shown on the initial data of the human intoxication FS. To compare the results obtained, we used the previously constructed models (model of a single-layer perceptron, model of a collective of neural networks) [4, 15, 18], where the initial data for training and testing were obtained by computer vision methods. Similarly, according to the topology and construction principles, we formed the models, the initial data for which were obtained using the given technology.

The architecture of the single-layer perceptron model is based on the well-known consequence of the Arnold-KolmogorovHecht-Nilsson theorem [19], according to which the maximum number of neurons in the hidden layer is limited by the right side of the expression:

$N_{h} \leq 2 \times N_{\text {in }}+1$,

где $N_{h}$ - number of hidden neurons, a $N_{i n}$ - number of input neurons. The neural network structure is shown in the following figure.

Thus, the neural network model contains 12 input neurons (according to the number of pupillogram parameters), 25 hidden layer neurons and 1 output neuron.

Creation of a neural network collective model (CoNN) is based on the combination of several models by the bootstrap method [20] and subsequent optimization of the structure based on the genetic algorithm [18]. Individual solutions generated at the output of each neural network are aggregated by a simple single-choice voting method.

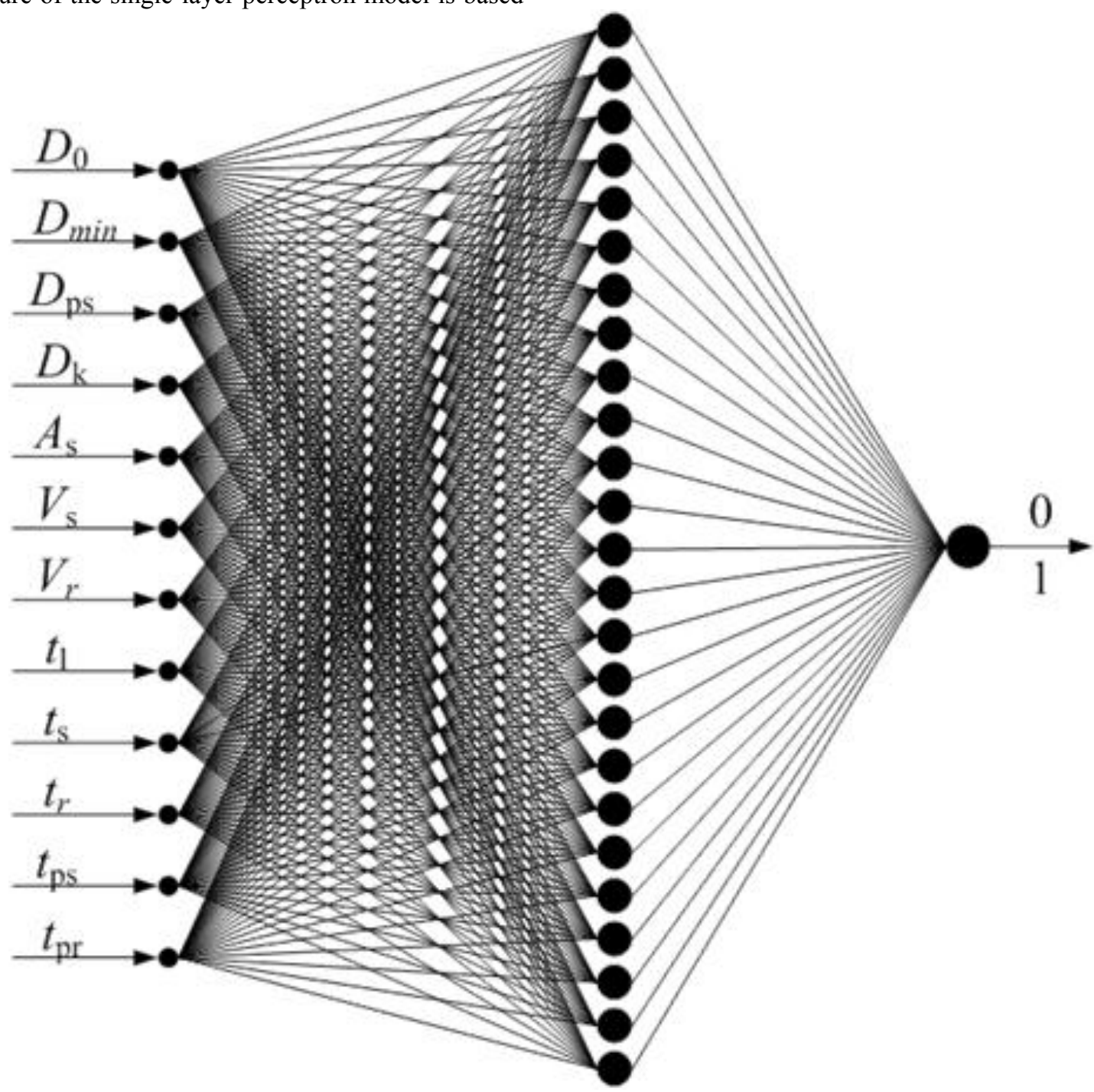

Figure 7. The structure of a single-layer perceptron model 
International Journal of Engineering Research and Technology. ISSN 0974-3154, Volume 13, Number 11 (2020), pp. 3616-3624

(C) International Research Publication House. https://dx.doi.org/10.37624/IJERT/13.11.2020.3616-3624

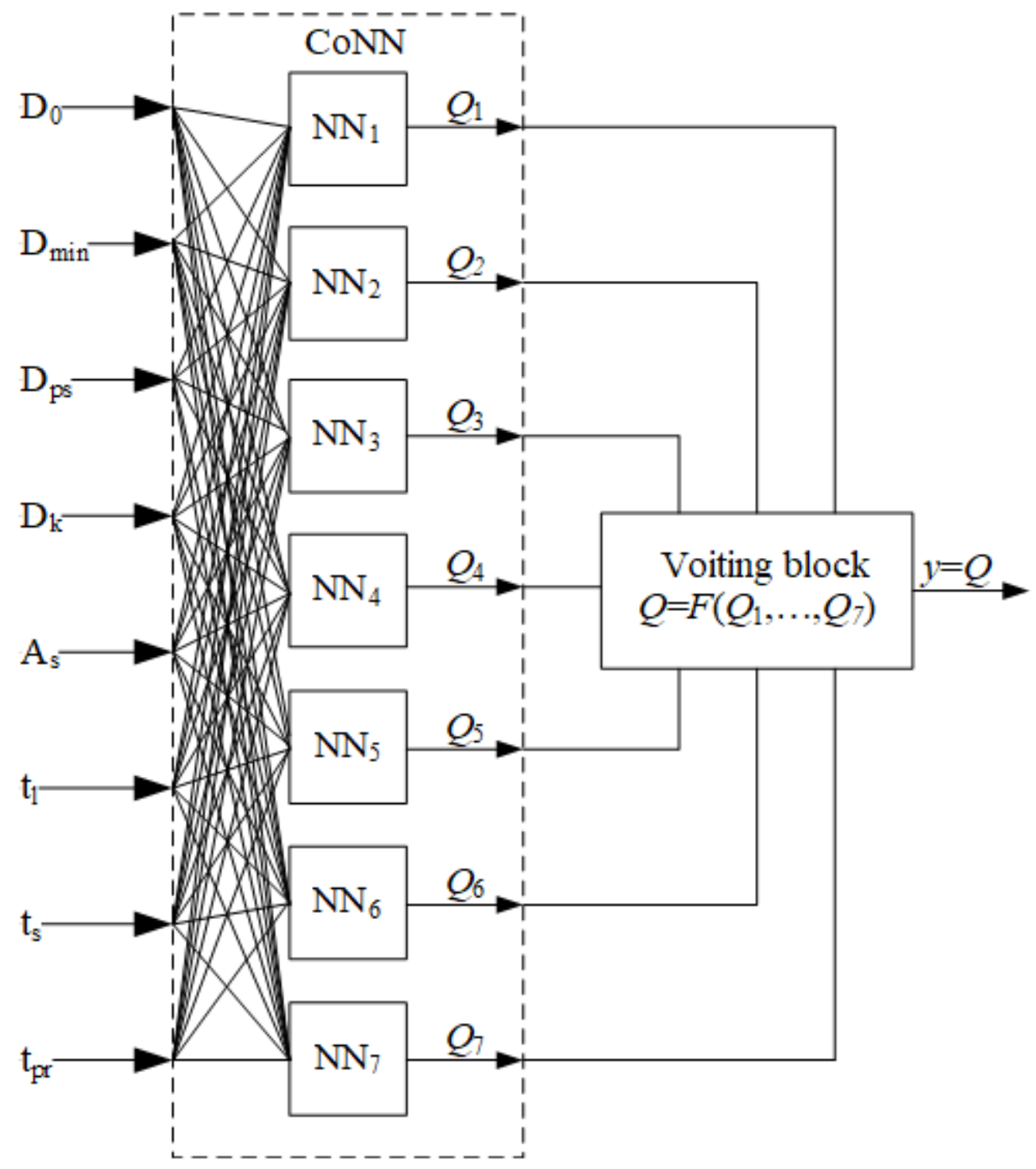

Figure 8. The structure of the neural network collective model

The CoNN model consists of 7 reduced neural networks with 10 hidden and 8 input neurons, the composition of which is determined by pupillometric parameters $D_{0}, D_{\min }$, $D_{\mathrm{k}}, D_{\mathrm{ps}}, A_{\mathrm{s}}, t_{\mathrm{l}}, t_{\mathrm{s}}$, and $t_{\mathrm{pr}}$. The complete mathematical model is presented in [18].

To assess the effectiveness of the developed models, we used type I and II errors, as well as the classification accuracy. The comparison results are given in the table.

Table 1. Comparison of the accuracy of different classification models

\begin{tabular}{|c|c|c|c|c|}
\hline Criteria & Method of obtaining circles & Type I errors & Type II errors & Classification accuracy \\
\hline Single layer perceptron ${ }^{1}$ & Computer vision & $0.8 \%$ & $5.2 \%$ & $94.0 \%$ \\
\hline Single layer perceptron ${ }^{2}$ & Manual marking & $0.7 \%$ & $5.1 \%$ & $94.2 \%$ \\
\hline CoNN model $^{1}$ & Computer vision & $0 \%$ & $3.3 \%$ & $96.7 \%$ \\
\hline $\mathrm{CoNN}_{\text {model }}{ }^{2}$ & Manual marking & $0 \%$ & $3.0 \%$ & $97.0 \%$ \\
\hline
\end{tabular}


As it follows from the given table, when using manual marking of circles, during the collection of initial data (models with index 2), the classification accuracy of the models is higher, and the number of type I and II errors is less, compared to models using computer vision methods to obtain the initial data (models with index 1). This indicates the achieved efficiency of the considered technology. Thus, the technology considered in the article makes it possible to more effectively solve the task of collecting initial data to create models for assessing the functional state of a person by pupillary response to changes in illumination.

\section{SUMMARY}

As the study showed, to collect initial data for creating models for assessing the functional state of a person by the pupillary response to a change in illumination, it is advisable to use the approach of manual marking of images of the required circles of the pupil and iris. The results of assessing the accuracy of the classification of the functional state of intoxication of a person on the basis of various classification models allow making a conclusion that the technology under consideration is the most preferable for creation of the intelligent data analysis models. It allows avoiding errors associated with the loss of data important for the analysis of the pupillogram properties, arising in the process of smoothing anomalies and noise. The classification accuracy based on the obtained initial data is higher than the classification accuracy of the models built on the initial computer vision data.

\section{CONCLUSIONS}

Thus, we solved the problem of collecting initial data for assessing the functional state of a person by his/her pupillary response to changes in illumination, which required the development of appropriate technology.

The developed technology for collecting initial data is based, on the one hand, on the traditional approach to obtaining pupillograms: video recording of human eyes at the time of pupillary reaction, but also introduces a criterion for improving the quality of initial data collection through the use of manual marking of the circles of the pupil and iris, which will allow using the obtained data to create adequate and highprecision mathematical models.

The technology considered in the article has also high efficiency due to the possibility of quickly collecting video fragments with pupillary reactions of the eyes of a group of persons.

The neural network model, built on the basis of the obtained initial data, demonstrated a high classification accuracy and a minimum level of errors of the first and second kind, compared to the models based on the initial data obtained using computer vision methods.

This indicates the effectiveness of the technology under consideration and the possibility of its practical use to create models for assessing the functional state of a person by the pupillary response to changes in illumination.

\section{ACKNOWLEDGEMENTS}

The work is performed according to the Russian Government Program of Competitive Growth of Kazan Federal University.

\section{REFERENCES}

[1] Lowenstein O. Electronic Pupillography, Why, How and When? The E. E. N. T. Monthly. 1959;38:549-558.

[2] Janisse MP. Pupillometry: The psychology of the pupillary response. - New York : Wiley, 1977.

[3] Velhover ES, Ananin VF. Introduction to Iridology: Pupillodiagnostics. - M. : Publishing House of the UDN, 1991: - 212 p.

[4] Akhmetvaleev AM, Katasev AS. Neural network model of human intoxication functional state determining in some problems of transport safety solution. Computer Research and Modeling. 2018;10(3):285-293.

[5] Akhmetvaleev AM, Katasev AS, Podolskaya MA. A neural network collective model and a software package for determining the functional state of a person. Caspian Journal: Management and High Technologies. 2018;1(41):69-86

[6] Akhmetvaleev AM, Katasev AS. Instrumental set of automation programs for determination of the functional state of a person. Automation of control processes. 2018;2(52):112-121.

[7] Skodras E, Fakotakis N. Precise Localization of Eye Centers in Low Resolution Color Images. IVC. 2015;36(1):51-60.

[8] Teikari P. Automated Pupillometry. Project work of measurement science and technology for the course "S108.3120 Project Work". - Helsinki University of technology centre for metrology and accreditation. Metrology research institute. 2007: P. 35.

[9] Alpern M, Ohba N. The effect of bleaching and backgrounds on pupil size. Vision Research. 1972;12(5): 943-951.

[10] Akhmetvaleev AM. Katasev AS, Shleimovich MP. The problem of stimulating the person's view direction in the tasks of contactless identification of potentially dangerous persons. Information security and personal data protection. Problems and ways to solve them: Materials of the VIII All-Russian Scientific and Practical Conference. - Bryansk: BGTU. 2016: P. 5-8.

[11] Akhmetvaleev AM. Algorithm of contactless identification of persons in a state of drug intoxication. XXII Tupolev Readings (School of Young Scientists): Materials of Reports of the International Youth Scientific Conference. - Kazan: Foliant Publishing House. 2015: 49-55.

[12] Kupriyanov AS. Methods of processing and analysis of pupillograms. University News. Instrument Making. 2009;8(52):58-63. 
[13] Kutsalo AL. Pupillometry as a method of express diagnostics of drug intoxication: thesis Pupillometry as a method of express diagnostics of drug intoxication: dis. of the candidate of medical sciences. - SPb. 2004: - 118 p.

[14] Kalnitskaya VE, Pogrebnoy AI. Binocular synchronous pupillometry in monitoring the functional state of highly qualified athletes. Physical culture, sport - science and practice. 2012;2:41-45

[15] Akhmetvaleev AM, Katasev AS. Mathematical support and software complex for determining the functional state of intoxication of a person by his/her pupillary response to light impulse exposure, Monograph. 2018.

[16] Viola P, Jones M. Robust real-time face detection. International journal of computer vision. 2004;57:137154.

[17] Katasev AS, Makarov DA. Methodology, algorithms and software for tracking a moving object in video surveillance systems. Bulletin of the Kazan State Technical University named after A.N. Tupolev. 2010;4: 145-150.

[18] Mustafin AN, Katasev AS, Akhmetvaleev AM, Petrosyants DG. Using Models of Collective Neural Networks for Classification of the Input Data Applying Simple Voting. Journal of Social Sciences Research. 2018;2018 (Special Issue 5): 333-339.

[19] Hecht-Nielsen R. Kolmogorov's mapping neural network existence theorem // IEEE First Annual International Conference on Neural Networks. - San Diego. 1987;3:1113.

[20] Efron B, Tibshirani R. Improvements on CrossValidation: The .632+ Bootstrap Method // Journal of the American Statistical Association. - Jun. 1997;92(438): 548-560.

Panishchev Oleg Yuryevich - Researcher of the Research Laboratory of Near Space Research of the Kazan (Volga) Federal University. In 2003, he graduated from Yelabuga State Pedagogical University with a degree in Physics, Informatics and Computer Science. Research interests: time series analysis, analysis methods of non-equilibrium distributed systems.

Babayev Rufat Faig Ogly - Junior Researcher of the Research Laboratory of Near Space Research of the Kazan (Volga) Federal University. In 2018, he graduated from the Kazan (Volga) Federal University with a degree in Radiophysics. Research interests: methods of mathematical modeling of physical processes, automation of scientific experiments.

Petrosyants Dmitry Gennadievich - graduate student of the Department of Information Security Systems of the Institute of Computer Technologies and Information Protection of the Kazan National Research Technical University named after A.N. Tupolev-KAI (KNRTU-KAI). In 2020, he received master's degree in Informatics and Computer Engineering at the KNRTUKAI. Research interests: data mining, computer vision, neural networks.

Katasev Alexey Sergeevich - Doctor of Technical Sciences, Professor of the Department of Information Security Systems of the Institute of Computer Technologies and Information Security of the Kazan National Research Technical University named after A.N. Tupolev-KAI (KNRTU-KAI). In 2002, he graduated from the Yelabuga State Pedagogical Institute with a degree in Physics, Informatics and Computer Engineering. In 2018, he received a master's degree at the KNRTU-KAI in the direction of Informatics and Computer Engineering. In 2019, he defended his doctoral thesis at the KNRTU-KAI. Research interests: data mining technologies, formation of knowledge bases of expert systems, neural network, fuzzy and neuro-fuzzy modeling.

Akhmetvaleev Amir Muratovich - Candidate of Technical Sciences, Associate Professor of the Department of Information Security Systems of the Institute of Computer Technologies and Information Security of the Kazan National Research Technical University named after A.N. Tupolev-KAI (KNRTU-KAI). In 2012, he received master's degree in Informatics and Computer Engineering at the KNRTU-KAI. In 2018, he defended his candidate thesis at the KNRTU-KAI. Research interests: intelligent time series analysis, neural networks, decision support systems, computer vision.

Akhmetvaleeva Irina Vladislavovna - master student of the Department of Information Security Systems of the Institute of Computer Technologies and Information Protection of the Kazan National Research Technical University named after A.N. Tupolev-KAI (KNRTU-KAI). She graduated from the KSU named after V.I. Ulyanov-Lenin with a degree in Mathematics. Research interests: data mining, expert systems.

Kataseva Dina Vladimirovna - graduate student, Senior Lecturer of the Department of Information Security Systems of the Institute of Computer Technologies and Information Protection of the Kazan National Research Technical University named after A.N. Tupolev-KAI (KNRTU-KAI). In 2008, she graduated from the Kazan State Financial and Economic Institute with a degree in Accounting, Analysis and Audit. In 2018, she received a master's degree at the KNRTU-KAI in the direction of Informatics and Computer Engineering. Research interests: intellectual analysis of time series, fuzzy logic, neural networks, decision-making support systems. 